INSTITUT NATIONAL DE RECHERCHE EN INFORMATIQUE ET EN AUTOMATIQUE

\title{
New Cross-Layer Channel Switching Policy for TCP Transmission on $3 G$ UMTS Downlink
}

\author{
Dinesh Kumar — Dhiman Barman — Eitan Altman — Jean-Marc Kelif
}

\section{$\mathbf{N}^{\circ}$ ????}

August 2006

Thème COM

apport

de recherche 



\title{
New Cross-Layer Channel Switching Policy for TCP Transmission on 3G UMTS Downlink
}

\author{
Dinesh Kumar, Dhiman Barman, Eitan Altman, Jean-Marc Kelif \\ Thème COM — Systèmes communicants \\ Projets Maestro
}

Rapport de recherche $\mathrm{n}^{\circ}$ ???? - August 2006 - 18 pages

\begin{abstract}
In 3G UMTS, two main transport channels have been provided for downlink data transmission: a common FACH channel and a dedicated DCH channel. The performance of TCP in UMTS depends much on the channel switching policy used. In this paper, we propose and analyze three new basic threshold-based channel switching policies for UMTS that we name as QS (Queue Size), FS (Flow Size) and QSFS (QS \& FS combined) policy. These policies significantly improve over a modified threshold policy in [1] by about $17 \%$ in response time metrics. We further propose and evaluate a new improved switching policy that we call FS-DCH (at-least flow-size threshold on DCH) policy. This policy is biased towards short TCP flows* of few packets and is thus a cross-layer policy that improves the performance of TCP by giving priority to the initial few packets of a flow on the fast DCH channel. Extensive simulation results confirm this improvement for the case when number of TCP connections is low.
\end{abstract}

Key-words: cross-layer, scheduling, resource, buffer management, DCH, FACH

Dinesh Kumar and Eitan Altman are at INRIA, BP 93, 06902 Sophia Antipolis, France. (\{dkumar, altman\}@sophia.inria.fr)

Jean-Marc Kelif is at France Telecom R\&D, 92794 Issy les Moulineaux, France. (jeanmarc.kelif@orange-ft.com)

Dhiman Barman, who is now at Computer Sc. \& Engg., University of California, Riverside, USA, carried out his part of this work during a visit to INRIA. (dhiman@ cs.ucr.edu)

The authors of this report are thankful to France Telecom R\&D who financially supported this work under the external research contract (CRE) no. 46130622.

* A flow is defined as a burst of packets in a TCP connection.

Unité de recherche INRIA Sophia Antipolis

2004, route des Lucioles, BP 93, 06902 Sophia Antipolis Cedex (France)

Téléphone : +334923877 77 - Télécopie : +33492387765 


\section{Nouvelle Politique Inter-Couche de Commutation pour Transmission TCP sur 3G UMTS Downlink}

Résumé : En 3G UMTS, deux canaux principaux de transport ont été crées pour la transmission de données sur le lien descendant : un canal commun FACH et un canal dédié DCH. Le performance de TCP en UMTS dépend beaucoup de la politique de commutation de canal utilisée. Dans cet article, nous proposons et analysons trois nouvelles politiques de commutations de canal pour l'UMTS basées sur des seuils simples que nous appelons politique QS (Queue Size), FS (Flow Size) et QSFS (QS et FS combinés). Ces politiques améliorent de manière significative (environ 17\%) le temps de réponse par rapport à la politique modifiée de seuil de [1]. De plus, nous proposons et évaluons une nouvelle politique de commutation, plus performante, que nous appelons politique de FS-DCH (atleast flow-size threshold on DCH). Cette politique favorise les connexions TCP courtes (i.e., de peu de paquets) et est ainsi une politique inter-couche qui améliore le performance de TCP en donnant priorité aux premiers paquets des connexions sur le canal rapide DCH. De nombreux résultats de simulation illustrent ce gain de performance dans le cas d'un petit nombre de connexions TCP.

Mots-clés : inter-couche, ordonnancement, ressource, gestion de buffer, DCH, FACH 


\section{Introduction}

Keeping in pace with the increasing demand from users for access to information and services on public and private networks, the third generation (3G) Universal Mobile Telecommunication System (UMTS) has been designed to offer services such as high speed Internet access, high quality image and video exchange and global roaming. Data traffic in UMTS has been classified broadly into four different classes, namely-conversational, streaming, interactive (e.g., web browsing) and background (e.g., email) classes. The bulk of data in streaming and interactive transmissions is carried over the downlink from UTRAN (UMTS Terrestrial Radio Access Network) to a UE (User Equipment). Data generated in the higher layers of UTRAN is carried over the air interface to the UEs via the downlink transport channels, which are mapped in the physical layer to different physical channels. There are two types of layer-2 downlink transport channels that have been provided in UMTS: dedicated channels and common channels. A common channel is a resource shared between all or a group of users in a cell, where as a dedicated channel is a resource identified by a certain code on a certain frequency and is reserved for a single user only. The only dedicated channel is termed as DCH and one of the six common transport channels that is mainly used for packet data on the downlink is the FACH channel [2]. The number of DCH channels in a UMTS cell is interference

limited. If a new user's connection cannot be admitted into the cell (this is decided by an appropriate interference based CAC or connection admission control), it must wait until a DCH channel is released by the already connected users or until when interference conditions become suitable for this new user to be allocated a new DCH channel. Being a dedicated channel, DCH guarantees higher data rates but the set-up time for DCH is significant (of the order of $250 \mathrm{~ms}$ ). On the other hand, the common channel FACH inherently guarantees lower data rates but its set-up time is less. According to the WCDMA (Wideband-CDMA) specifications detailed by the 3GPP group, for a particular user, long flows with large amount of packets can be transmitted on the user dedicated DCH channel and short flows of few packets can be transmitted on the common FACH channel which is shared by all users. However, the 3GPP specifications do not provide any standardization of such a channel selection/switching policy. A network operator is free to choose its own proprietary channel switching policy.

\subsection{Main Contributions}

In this paper, we propose some new basic channel switching policies and analyze their performance characteristics through simulations. These new policies are based on an extension of the modified threshold policy in [1]. In the simulation results (Section 5] we observe that our new switching policies improve on the modified threshold policy in [1] by around $17 \%$ in response time metrics. Based on some observations about the $\mathrm{DCH}$ and $\mathrm{FACH}$ channel characteristics and the need for distinction of long and short TCP flows, we further propose another new cross-layer channel switching policy, which is our main contribution in this paper. To the best of our knowledge, ours is the first attempt to propose such a cross-layer channel switching policy for UMTS downlink that is based on diffrentiation between long and short TCP flows. All the new policies are in accordance with the current WCDMA specifications and we evaluate their performance in terms of response time and slowdown using simulations. 


\subsection{Synopsis}

We start in Section 2 by defining the basic threshold-based channel switching policies. We name them as QS (Queue Size) policy, FS (Flow Size) policy and QSFS (QS \& FS combined) policy. In all these policies a new flow or connection starts on the common FACH channel. In Section 3 we observe that in the basic policies the switching delay for connections switching from FACH to DCH and vice-versa is not very significant as compared to the transmission time of packets on the FACH channel, given the fact that FACH is a low bandwidth channel with high priority signaling traffic on it. We also argue that it is advantageous for short flows to have small response times. This observation and argument motivates us to propose the design of a new cross-layer policy that we call FS-DCH (at-least flow-size threshold on DCH) policy in which we try to achieve better response time and slowdown for short flows. In Section 4 we describe the network model and simulation set-up that we have used for performance evaluation of all the policies. Section 5 leads to discussion on the various observations that can be made from simulation graphs obtained. We finally conclude in Section 6

\subsection{Related Work}

Most of the existing channel switching policies are very simple, timer and threshold based policies and do not involve any complex or cross-layer switching criteria. Queue size threshold based policies have been proposed in [1] in which a new connection is initially allocated to FACH. On indication that the current flow of the connection might be long (i.e., a long buffer queue for that source is observed), then beyond some upper threshold, the Packet Scheduler in UMTS tries to allocate a $\mathrm{DCH}$ to that connection (if one is available). While on DCH, when the queue size of the connection falls below another lower threshold, the connection is switched back to FACH. The authors in [1] also present a modified threshold policy, in which, while a connection is on $\mathrm{DCH}$, if its queue size falls below a lower threshold, a timer is started and the connection remains on DCH. If there are no arrivals during the timer period, the connection is switched back to FACH. The timer is used to let the TCP acknowledgements (ACKs) reach the sender and release new packets. In [3], the switching policy switches connections from FACH to DCH when the number of packets transmitted (i.e., flow size) for a given user on FACH exceeds a threshold. The choice of the threshold depends on the load on FACH and other QoS conditions. In [5], a switching policy based on bandwidth demand has been proposed. A connection is switched from FACH to DCH if its bandwidth demand exceeds a threshold and remains on FACH otherwise. The channel switching schemes in [4] work with blocking and unblocking packets present in the RLC (Radio Link Control) and MAC sub-layers and different schemes propose to transmit the unblocked packets on either common or dedicated channels, differently.

The authors in [1] have implemented a PS+FCFS queue system, i.e., the FACH channel uses a processor sharing (PS) scheduling mechanism and the DCH channel uses an FCFS scheduling mechanism. However, the policies that we propose in this paper are different from those proposed by the authors in [1], [5] and [6], since we use PS+Priority and $\mathrm{LAS}^{1}+$ Priority queue systems for

\footnotetext{
${ }^{1}$ LAS: Scheduling based on Least Attained Service
} 
FACH and DCH channels and not PS+FCFS queue systems. This will become more clear in the following section.

\section{Basic Channel Switching Policies}

We propose three new basic threshold-based channel switching policies for packet data transmission on the downlink of a single UMTS cell. They are based on an extension of the modified threshold policy in [1]. In all these policies, the FACH channel is served with either a PS or a LAS scheduling mechanism and the DCH channel is implemented as Priority scheduling with priority given to connections having maximum queue lengths. Before we discuss in detail about the three channel switching policies, we define below the notations used in their formal definitions:

- Let $Q(i)$ denote the queue length of a connection $i$ at the UMTS base station (NodeB).

- Let $T_{h}$ and $T_{l}\left(T_{h} \geq T_{l}\right)$ denote two thresholds on the queue length $Q(i)$ when the connection is on FACH and DCH channels, respectively.

- Let $f(i)$ denote the cumulative flow size (i.e., number of packets transmitted) over the FACH and DCH channels, for the current flow of a connection $i$.

- Let ' $s$ ' denote a threshold on the cumulative flow size $f(i)$ of the current flow.

- In all the policies described in this paper, a connection starts on FACH by default and then if a DCH is available, it is switched to DCH depending on different thresholds. If a DCH is not available then a request $r_{i}$ corresponding to this connection $i$ is added to a request set so that later when a DCH is available, connection $i$ will be switched to DCH. Let $R$ denote this request set.

- Let $W(i)$ denote the total time for which a request $r_{i}$ of connection $i$ remains unserved. Alternatively, it denotes the total time for which a connection $i$ has been waiting to be switched to DCH since its request $r_{i}$ to switch to DCH was added to $R$.

- Let $N_{d c h}$ denote the total number of DCH channels in a single UMTS cell.

- Let $U_{d c h}$ denote the total number of DCH channels that have been allocated or currently in use in the UMTS cell. Note that $U_{d c h} \leq N_{d c h}$.

\subsection{QS Policy}

In the QS (Queue Size) policy with parameter $T_{h}$, a new connection $i$ starts on the FACH channel and waits for its queue length to exceed an upper threshold $T_{h}$ before switching to DCH. If there is no $\mathrm{DCH}$ channel available then a request $r_{i}$ for this connection to switch to $\mathrm{DCH}$ is made. For a connection $j$ on DCH when its queue length drops below the lower threshold $T_{l}$, a timer is started for $T_{\text {out }}$ seconds. If there are packet arrivals during the timer period, the timer is reset. When the timer expires, if the queue length of connection $j$ is still below the lower threshold and another 
set of connections on FACH are attempting to switch to DCH and no more free DCH channels are available, the connection $j$ switches back to FACH. Once this connection switches to FACH after a switch delay (of around $250 \mathrm{~ms}$ ), a connection having the maximum queue length among the set of connections on FACH that were attempting to switch to DCH, is switched to DCH. In this way we give priority to the connections with the maximum queue lengths while switching from FACH to DCH. This is what we mean by Priority scheduling on the DCH channel. PS+Priority then implies that, FACH uses PS scheduling mechanism and DCH uses Priority scheduling. This PS+Priority queue system is the essential difference between our new basic QS, FS and QSFS policies and the policies proposed in [1] which use PS+FCFS queueing. We will see later in Section 5 that our new policies significantly improve over the modified threshold policy in [1] by around $17 \%$ in response time metrics. This leads to the conclusion that PS+Priority queueing system is the main feature due to which our new policies improve over the modified threshold policy in [1]. The QS policy can be formally defined as follows:

QS policy: The QS (Queue Size) policy is characterized by the following set of rules:

- A connection $i$ starts on FACH by default. It switches to DCH if $Q(i)>T_{h} \&$ $U_{d c h}<N_{d c h}$.

If $Q(i)>T_{h} \& U_{d c h}=N_{d c h}$ then $r_{i}$ is added to $R$.

- If connection $i$ is on DCH then if $Q(i)<T_{l}$, a timer is started for duration $T_{\text {out }}$ seconds. If there are packet arrivals during the timer period, the timer is reset. When the timer expires and $Q(i)<T_{l}$, then, if (1) $U_{d c h}=N_{d c h} \& R \neq \phi$ then connection $i$ switches to FACH and connection $j$ with $r_{j} \in R$ switches to DCH, where connection $j$ is chosen such that

$$
r_{j}=\arg \max _{r_{k} \in R} Q(k),
$$

else, (2) the connection $i$ remains on DCH and another timer of duration $T_{\text {out }}$ seconds is started.

In the above definition, once connection $j$ switches to DCH successfully, $r_{j}$ is deleted from the request set $R$. The motivation behind QS policy is to treat short flows and long flows differently. The size of a flow can be estimated by its queue size. Short flows will not exceed a sufficient upper threshold $T_{h}$ on the queue size and will get served on FACH. Thus, the idea is to avoid switching cost for short flows as the cost may be more or comparable to the service requirement of the short flows. Large-sized or long flows on the other hand will see their buffer queue build-up and will be switched to DCH. An important advantage of this policy is that using only local information (i.e., queue size) which is easily available, implicit queue size based scheduling can be implemented in a scalable (with number of users) fashion. 


\subsection{FS Policy}

In the FS (Flow Size) policy with parameter ' $s$ ', the Packet Scheduler waits for the number of packets served for the current flow of a connection to exceed a threshold ' $s$ ' before switching it to DCH. A connection on DCH switches back to FACH according to the same rule as in QS policy. The FS policy can be formally defined as follows:

FS policy: The FS (Flow Size) policy is similar to the QS policy except for the fact that a flow size threshold ' $s$ ' is used instead of the queue size threshold $T_{h}$ on FACH. It is thus characterized by the following set of rules:

- A connection $i$ starts on FACH by default. It switches to DCH if $f(i)>s \&$ $U_{d c h}<N_{d c h}$.

If $f(i)>s \& U_{d c h}=N_{d c h}$ then $r_{i}$ is added to $R$.

- If connection $i$ is on DCH then it follows the same rule as in QS policy. When connection $i$ switches to FACH successfully, $f(i)$ is set to 0 .

The FS policy is similar to QS policy except for the fact that the flow size is directly computed from the number of packets served. A flow gets threshold amount of service on FACH, exceeding which the flow is termed as a long flow and switched to DCH. The policy is scalable with number of users as the size of a flow can be computed locally.

\subsection{QSFS Policy}

In QSFS (QS \& FS combined) policy a connection on FACH switches to DCH when conditions of both QS and FS policy are satisfied. A connection on DCH switches back to FACH according to the same rule as in QS policy. The QSFS policy can be formally defined as follows:

QSFS policy: In this policy, we combine the QS and FS policies. It is thus characterized by the following rules:

- A connection $i$ starts on FACH by default. It switches to DCH if $Q(i)>T_{h} \&$ $f(i)>s \& U_{d c h}<N_{d c h}$.

If $Q(i)>T_{h} \& f(i)>s \& U_{d c h}=N_{d c h}$ then $r_{i}$ is added to $R$.

- If connection $i$ is on DCH then it follows the same rule as in QS policy. When connection $i$ switches to FACH successfully, $f(i)$ is set to 0 .

We defer the performance evaluation through simulations of the above mentioned policies to Section 5

$R R n^{\circ} 0123456789$ 


\section{Designing a New Cross-Layer Channel Switching Policy}

Most of the software applications running over UTRAN use TCP as the transmission protocol. TCP reacts to congestion and losses either by drastically reducing its congestion window size after a timeout, or with some fluidity through fast retransmit procedures. For short flows with small number of packets, a loss of one of the last few packets is often detected only after a timeout, due to insufficient NACKs received by the sender. Thus timeouts of short flows are not very effective in reducing network congestion and one of the most important aspects on the downlink channel is to sustain efficient TCP performance by preventing timeouts of short flows and congestion in buffer queues [7|. For example in peer-to-peer file exchanges, two users exchange a small number of packets (generating short flows) before one of them downloads a long heavy data file. Same is true for FTP and HTTP web browsing traffic where packet exchanges between applications running across UTRAN and a UE consist either entirely of short flows (if caching is enabled in the browser) or of short flows followed by a long file transfer (if caching is not enabled). Similarly, short flows are also generated by conversational voice packet transfers (not streaming voice) where maximum acceptable end-to-end delay according to the human perception is around $400 \mathrm{~ms}$. Thus from user ergonomics point of view, it would seem advantageous to minimize the transfer times of short flows by giving them priority over long flows and serving them on a faster link [7]. This motivates us to design a cross-layer channel switching policy in which the initial packets of a TCP flow are given priority on a fast link and if this flow turns out to be a long flow then it can be afforded to serve this flow on a slow link unless it builds up a very large queue length on the slow link, in which case this long flow needs to be switched back to the fast link.

In all the existing and basic channel switching policies discussed previously in Sections 1.3 and 2 respectively, a new flow of a connection always starts on the slow FACH channel and waits until some threshold parameter has been attained, before switching to the fast $\mathrm{DCH}$ channel. For short data bursts of say less than 10 packets, it may take a long time (on slow FACH) for them to surpass any threshold parameter or they may never surpass it at all (due to insufficient number of packets). On the other hand, long flows with a large number of packets will most probably surpass the thresholds and get a chance to be transmitted on the fast DCH channel. Thus there is a possibility that short flows in their entirety will suffer high transmission times on the slow FACH channel, where as for long flows even though their initial few packets are transmitted on the FACH channel, their overall transmission time may improve since most of their (remaining) packets are transmitted on the DCH channel. This intuition can be further strengthened by some concrete calculations that follow.

Let us take a closer look on the FACH channel. The FACH channel has a very low set-up time, usually has a capacity of around $33 \mathrm{kbps}$ and has a high priority signal traffic (from a constant bit rate (CBR) source) running on it apart from the data packets. The CBR source transmits signal traffic at the rate of around $24 \mathrm{kbps}$. So a short data burst of say 10 packets of 1 kbyte each will take approximately 8.88 seconds (or 2.42 seconds in the best case when CBR traffic is absent) to be transmitted on the FACH channel. Now let us consider the DCH channel. The DCH channel has a capacity of around $384 \mathrm{kbps}$. There is a set-up time of around $250 \mathrm{~ms}$ for the DCH channel which is much higher than the set-up time of the FACH channel. So unlike the mechanism used in existing and basic switching policies, if a connection starts on FACH and switches to DCH immediately 
without waiting to attain any threshold, a 10 kbytes burst will get transmitted in approximately $0.25+10 \times 8 / 384=0.25+0.208=0.458$ seconds. This significantly reduces the transmission time by a factor of about 20 in the presence of CBR traffic and about 5 in its absence. Thus, switching a new flow to DCH as soon as it starts can be beneficial for short data bursts which would have otherwise suffered high transmission times on the slow FACH channel. This clearly illustrates that the existing and basic policies discussed previously in Sections 1.3 and 2 respectively, suffer from a major drawback. The drawback being that a new flow is allowed to transmit initially on slow FACH for a long time (by the threshold mechanism) before it gets a chance to be transmitted on the fast $\mathrm{DCH}$.

The above argument gives us the motivation to design a cross-layer channel switching policy in which the initial few packets of a new TCP flow of a connection on FACH are given priority on the fast $\mathrm{DCH}$ channel by switching the connection from $\mathrm{FACH}$ to $\mathrm{DCH}$ as soon as possible. If this new flow is a short flow then it will be entirely served on DCH thus ensuring minimum transfer times for short flows, as explained with the help of some calculations in the previous paragraph. Otherwise if this flow turns out to be a long flow, then later if the buffer queue length of the associated connection falls below a threshold $T_{l}$, the connection is either preempted and switched back to FACH to allow other new flows on $\mathrm{FACH}$ to switch to $\mathrm{DCH}$, or the connection remains on $\mathrm{DCH}$ and then ultimately times out (in the absence of packet arrivals during an inactivity timer period) and is switched to FACH indicating the end of current flow on the connection. Thereafter, any new packet arrivals on this timed out connection on FACH will be termed as a new flow. Thus at any given instant there are either new flows on FACH attempting to switch to $\mathrm{DCH}$, or there are old flows on FACH (which may also be long with a high probability) which have already transmitted their initial few packets (say at least first ' $s$ ' packets) on DCH. If the buffer queue length of the connections with old flows surpasses the threshold $T_{h}$, then they attempt to switch to DCH again in order to minimize the use of FACH channel, since it is a very slow channel that can cause significant increase in transmission times.

Note that in our new policy described above, a new connection must always necessarily start transmitting on the common FACH channel, since the number of DCH channels are interference limited and a DCH may not always be available to be allocated for a new connection. When a connection $i$ on FACH attempts to switch to $\mathrm{DCH}$ and if no DCH channel is available, a request $r_{i}$ to switch to $\mathrm{DCH}$ is pushed into a request set $R$ and this request is served when a DCH channel is available later.

We call the strategy of allowing a new flow to transmit at least its first ' $s$ ' packets on DCH as the first ' $s$ ' on DCH mechanism and it is one of the two key features of our new improved switching policy. The other key feature is the use of dual-level priority switching mechanism. This mechanism works as follows. If more than one connections on FACH are candidates (i.e., they have requested to switch to $\mathrm{DCH}$ ) to be switched to a single available DCH channel, then the dual-level priority switching mechanism chooses only one connection among all connections with new flows, on a first-come first-served (FCFS) basis, to be switched to DCH. In the absence of connections with new flows, the connection with the maximum queue length among all connections with old flows, is switched to DCH. We term our cross-layer channel switching policy as FS-DCH (at-least flow-size threshold on DCH) policy and it can be formally defined as follows: 
FS-DCH policy: The FS-DCH policy is defined as follows:

- A connection $i$ starts on FACH by default. A connection switches to DCH if (1) $f(i) \leq s \& U_{d c h}<N_{d c h}$ or (2) $f(i)>s \& Q(i)>T_{h} \& U_{d c h}<N_{d c h}$.

If (1) $f(i) \leq s \& U_{d c h}=N_{d c h}$ or (2) $f(i)>s \& Q(i)>T_{h} \& U_{d c h}=N_{d c h}$ then $r_{i}$ is added to $R$.

In this rule, the condition (1) causes a new connection starting on FACH to attempt to switch to $\mathrm{DCH}$ as soon as possible.

- If connection $i$ is on DCH and $Q(i)<T_{l}$ then

(a) if $f(i) \leq s$, then it follows the same rule as in QS policy. When connection $i$ switches to FACH successfully, $f(i)$ is set to 0 .

(b) if $f(i)>s$, then

if (1) $U_{d c h}=N_{d c h} \& R \neq \phi$, connection $i$ is preempted and it switches to FACH and connection $j$ with $r_{j} \in R$ switches to $\mathrm{DCH}$, where connection $j$ is chosen such that $f(j) \leq s$ (its a new flow) and

$$
r_{j}=\arg \max _{r_{k} \in R} W(k) .
$$

If there is no such connection that satisfies the condition $f(j) \leq s$ then connection $j$ is chosen such that $f(j)>s$ (its an old flow) and

$$
r_{j}=\arg \max _{r_{k} \in R} Q(k),
$$

else, (2) it follows the same rule as in QS policy.

In the above definition, once connection $j$ switches to DCH successfully, $r_{j}$ is deleted from the request set $R$. We defer the performance evaluation through simulations of the FS-DCH policy to Section 5

\section{UMTS Network Model \& Simulation Setup}

In this section we describe the UMTS network model that we use for performance evaluation of the various aforementioned policies through simulations. The model described here is very similar to the one in [1]. We consider a network model with $N_{t c p}$ TCP sources which need to send data to mobile receivers. We assume a single cell scenario with one NodeB base station and several mobile stations which act as destinations for TCP traffic. The TCP sources are assumed to be connected to the base station of the cell with a high speed (5mbps, 30ms) link. The base station can transfer data from a TCP source on either DCH or FACH at a given time. There is one FACH and $N_{d c h}$ DCH channels in the system. The FACH is a time division multiplexed channel. In addition to any TCP 


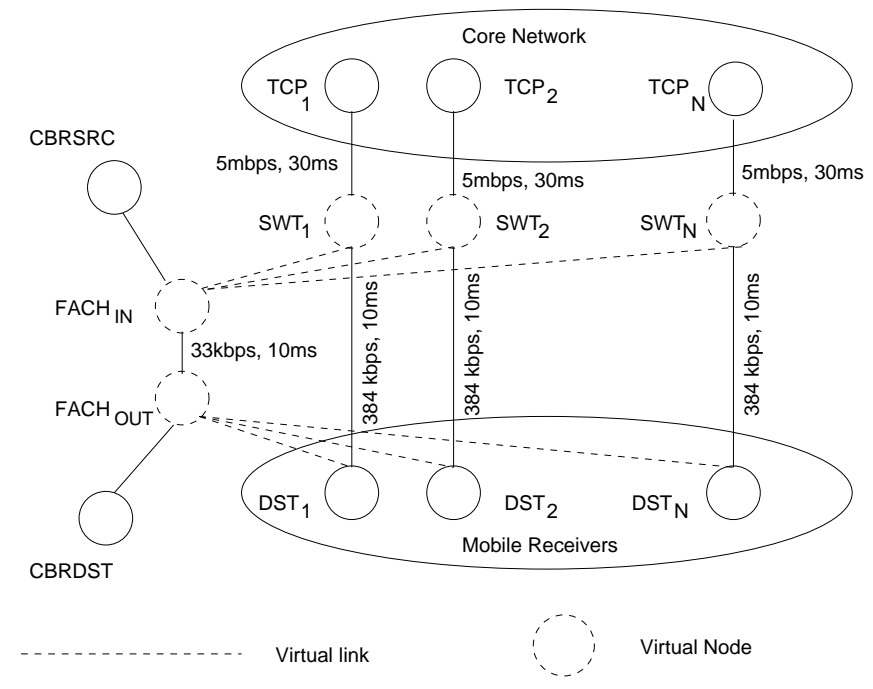

Figure 1: Simulation Setup

connections which may be present on a $\mathrm{FACH}$, there is signaling traffic which must be transmitted on the FACH. The signaling traffic has priority over the TCP connections. During the silence periods of the signaling traffic, data from one or more TCP connections can be transmitted on the FACH. Data from the TCP connections is assumed to be transmitted on the FACH with a PS or LAS service mechanism. If all the DCHs have a TCP connection allocated, a connection on DCH should be first switched to FACH before a connection from FACH can be switched on to a particular DCH. This means that a switch can take up to $500 \mathrm{~ms}$ (if there is already a TCP connection configured on the $\mathrm{DCH}$ and if we consider the connection release time to be the same as the connection set-up time). Switching from one channel to another is costly in time and signaling. In the model we assume that there exists a queue corresponding to each TCP connection in the NodeB base station. The base station is hence able to track both the queue length and the number of packets served (flow size) for each connection. During the switching time from one channel to another, no packets from the queue of the TCP connection being switched can be transmitted. While a connection is switching from one channel to another, the ACKs of a TCP connection traverse the original channel until the switch is completed.

The simulation setup for the above described network model is presented in Figure 1 Each TCP source node $T C P_{i}$ is connected to a routing node called Switch $\left(S W T_{i}\right) . S W T_{i}$ is present inside the NodeB base station and can be connected either to the $F A C H_{I N}$ or directly to the TCP destination via the $\mathrm{DCH}$. The $S W T_{i}$ node has been introduced to simplify the simulations and may not be present inside a real NodeB base station. The $F A C H_{I N}$ is another virtual node which simulates either the PS or LAS service discipline taking place on the FACH. In the PS discipline, the node $F A C H_{I N}$ gives priority to the traffic from $C B R S R C$ while serving the packets from the $S W T_{i}$ 's (only those which are currently not transmitting on $\mathrm{DCH}$ ) in a round-robin manner. 
We note that there are no queues at $F A C H_{I N}$ and all the packets are either queued at $S W T_{i}$ or at the $C B R S R C$. The $C B R S R C$ simulates a constant bit rate source of signaling/control traffic. It generates packets at rate $R_{s i g}$ and is assumed to be present within the NodeB. The signaling traffic flows from the NodeB to the mobile receivers. Even though we model the destination of the signaling traffic $(C B R D S T)$ as another node different from the mobile destinations $D S T_{i}$, we note that it does not affect the simulations as simultaneous transfer of data and control packets to the same mobile receiver is indeed possible in UMTS when different channels are used. The links $S W T_{i}-$ $F A C H_{I N}$ are virtual links within the base station and thus have zero delay. Note that the data from $S W T_{i}$ to $D S T_{i}$ can take two different routes i.e., $S W T_{i}-F A C H_{I N}-F A C H_{O U T}-D S T_{i}$ (via $\mathrm{FACH}$ ) or simply $S W T_{i}-D S T_{i}$ (via DCH). At any given time only one of the above two routes can be active for a given connection. Although in the simulation scenario we have as many DCH links as TCP source nodes, the simulation allows us to activate not more than $N_{d c h}$ DCH channels at a time, which may be chosen strictly smaller than the number of TCP sources $\left(N_{t c p}\right)$. In the simulations we switch over from FACH to DCH by changing the cost of the links and recomputing the routes. This is done as follows. Initially, the cost of direct path from the Switch to the TCP destination is set to 10 and the cost of all other links to 1 . Hence, the traffic gets routed through the FACH. When a switch is required, the cost of DCH is set to 1 and the routes are recomputed. This activates the $\mathrm{DCH}$ and the traffic gets routed on the DCH.

\subsection{Limitations and Assumptions}

The layer 2 in UTRAN consists of two sub-layers: MAC layer and RLC (Radio Link Control) layer. As described previously, the physical layer (layer 1) offers services to the MAC layer via transport channels of two types: dedicated channels and common channels. The MAC layer in turn offers services to the RLC layer above it through logical channels. The different logical channels are mapped to the transport channels in the MAC layer. The two most important logical entities in MAC layer are MAC-c/sh and MAC-d. The MAC-c/sh entity handles data for the common and shared channels, where as the MAC-d entity is responsible for handling data for the dedicated channels. However, the execution of switching between common and dedicated channels is also performed by the MAC-d entity in UTRAN (in the serving RNC) based on a switching decision derived by the channel switching algorithm that resides in the RRC (Radio Resource Controller).

Data packets or SDUs (Service Data Units) arriving from upper layers are segmented into smaller data packets or PDUs (Protocol Data Units) by the RLC layer and PDUs are then forwarded to the MAC layer. In our network model used to carry out the simulations for performance evaluation of various switching policies, we do not consider the segmentation of SDUs into PDUs. In other words, we do not model the RLC layer since the main focus of this paper is to investigate the channel switching mechanism. We thus model only the MAC-d entity in the MAC layer. We also do not take care of packet loss, mobility and handovers, since considering them would highly complicate the model and it is beyond the scope of this paper. 


\subsection{Simulation Parameters}

We use ns-2 [8] in order to simulate the various switching policies for performance evaluation. The simulation parameters used are described below:

- We consider 2 cases for the number of dedicated channels, $N_{d c h}$ equal to 1 and 2 . We vary the number of TCP sources as $N_{t c p}=2,3,5$ and 10 .

- The duration of simulations is taken to be 200,000 secs. in order to reach stationarity.

- The transmission rates for FACH and DCH channels are considered to be $33 \mathrm{kbps}$ and 384 kbps, respectively.

- The switching cost $D_{s w}$ (in terms of time) between FACH and DCH channels is $250 \mathrm{~ms}$.

- We consider the signaling traffic source (non TCP traffic source) that uses the FACH, to be a constant bit rate CBR source with rate $R_{\text {sig }}=24 \mathrm{kbps}$. It sends a 1 kbyte packet at an interval of $1 / 3$ s and has a non preemptive priority over TCP traffic.

- The TCP connection traffic model is as follows: In a TCP connection, data arrives in bursts. The number of packets in a burst has a Pareto distribution and the shape parameter is taken to be $k=1.1$. The average file size is taken to be $F S_{a v g}=30$ kbytes. A TCP connection alternates between "ON" and "OFF" states. The ON state is comprised of several bursts and no packets are transmitted during the OFF state. In the ON state, the inter-arrival time between successive bursts is exponentially distributed with mean $T_{O N}=0.3 \mathrm{~s}$. At the end of each burst in ON state, the connection goes into OFF state with probability $P_{O F F}=0.33$. It remains in the OFF state for an exponentially distributed duration with mean $T_{O F F}=5 \mathrm{~s}$ before it goes back into $\mathrm{ON}$ state again.

- The value of $T_{l}$ (lower threshold on DCH) is taken as 1 and the packet size as 280 bytes.

\section{Performance Evaluation of Policies}

In this section, we analyze the results obtained from an extensive set of simulations of the various channel switching policies that we have discussed until now. We study PS scheduling of TCP sources on the FACH channel for QS, FS, QSFS and FS-DCH policies. In addition to this we also study LAS scheduling on FACH channel for FS policy specifically. LAS scheduling can also be studied with other policies, but since LAS looks at the number of served packets, which relates to the flow size, FS policy is the most appropriate one to study with LAS scheduling.

In Figures 25. we compare different policies in terms of response time and slowdown as a function of $s$ or $T_{h}$, as the case may be. The response time is calculated as the total average time required to completely transmit a burst. By completely transmitting a burst, we mean the time until a TCP ACK for the last packet of a burst sent, is received at the sender side. Slowdown is defined as the response time divided by the average burst size. So, for an average burst size of $x$, if $T(x)$ is its response time then the slowdown $S(x)$ is defined as $\frac{T(x)}{x}$. In Figure 2 a), we observe that FS-DCH 


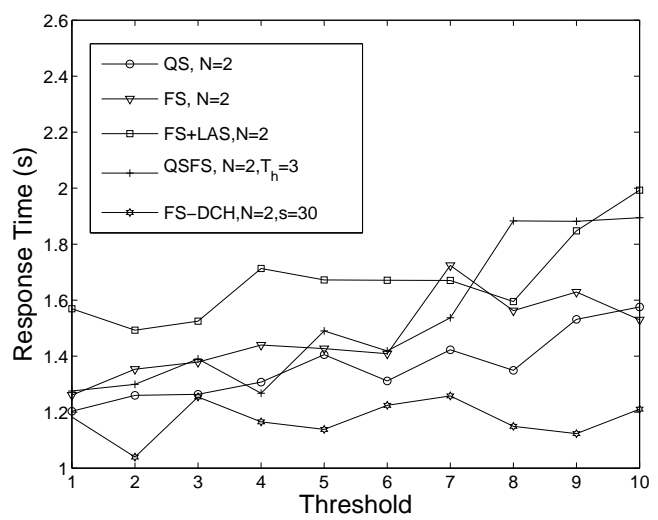

(a)

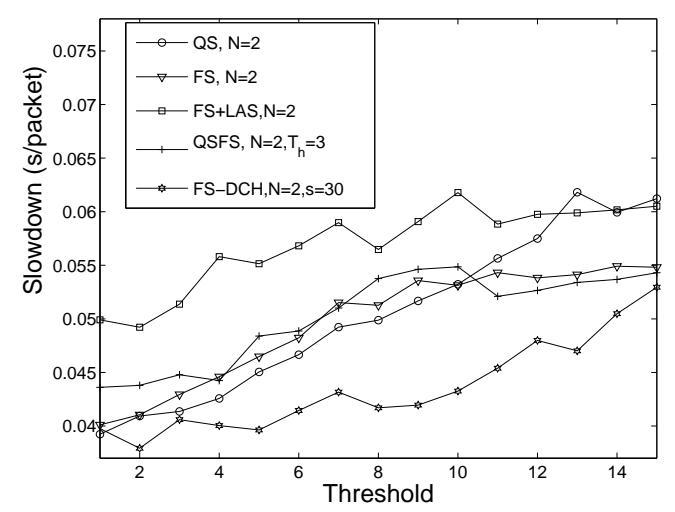

(b)

Figure 2: Comparison of different policies in terms of response time and slowdown metrics for $N_{t c p}=2, F S_{a v g}=30$ kbytes and $N_{d c h}=1$.

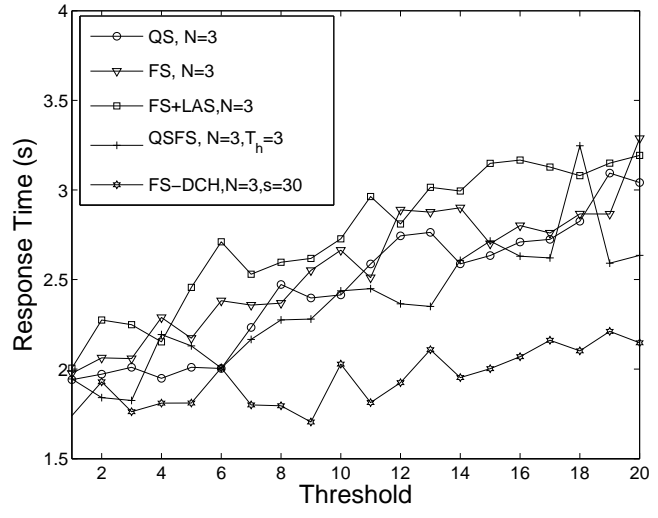

(a)

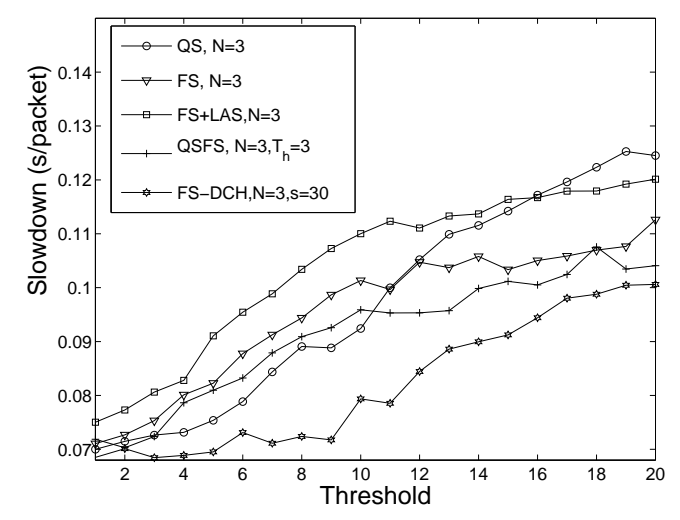

(b)

Figure 3: Comparison of different policies in terms of response time and slowdown metrics for $N_{t c p}=3, F S_{a v g}=30$ kbytes and $N_{d c h}=1$. 
outperforms all other policies in terms of response time, where as FS+LAS scheme has the highest response time. The other three schemes have comparable response times. The average improvement in response time achieved by FS-DCH over all other policies is around $30 \%$. Within the range of threshold values shown, we observe an increasing trend in response time under all policies except for FS-DCH. The QS policy performs slightly better than the FS policy in minimizing mean response time.

Under QS, FS and QSFS policies, at higher values of $T_{h}$ an increase in the response time is observed because a higher value of $T_{h}$ implies more time is spent in the FACH. The FACH is a low bandwidth channel which has high priority signaling traffic on it. This results in low average bandwidth being shared amongst the TCP connections due to the following reason. For a TCP connection, the switch to DCH is based on its current buffer size which in turn depends on its current congestion window size. The congestion window size is incremented whenever an ACK is received by the sender. When a TCP connection is on a low bandwidth link, the window builds up slowly due to delay in receiving an ACK. This slow buildup of the window size results in slow buildup of the current buffer size. As the value of $T_{h}$ is increased, a TCP connection has to spend more time on the slow FACH, resulting in a higher delay.

The comparison of average slowdown in Figure 2 b) shows that the slowdown metric follows the same trend as that of average response time. FS-LAS has the highest slowdown and FS-DCH has the lowest. Other policies perform almost the same except that performance of QS worsens for higher values of the threshold.

From the above discussions it can be concluded that the cross-layer FS-DCH policy is better than all other policies for $N t c p=2$.

In Figure 3 we plot the average response time and slowdown for $N_{t c p}=3$. It can be easily seen that FS-DCH again performs the best in terms of both response time and slowdown and all other policies perform comparably among themselves. The average improvement in response time achieved by FS-DCH over all other policies is around $36 \%$.

In Figure 4 we plot the average response time and slowdown for different policies for $N_{t c p}=5$, where we can easily see that the performance metrics are very different from those observed for $N_{t c p}=2$ and 3. Here, on an average, QS achieves the lowest response time and slowdown instead of FS-DCH. The improvement of QS over all other policies in response time metrics is on an average $18 \%$. FS-LAS gives the highest response time and slowdown and FS-DCH is worse than QSFS in terms of both response time and slowdown.

In Figure 5 for $N_{t c p}=10$, we observe that on an average, QSFS policy performs the best in terms of both response time and slowdown. The average improvement of QSFS policy over other policies in response time metrics is around $21 \%$. For threshold values $>12$ packets, the response times of QS and FS+LAS are comparable.

As mentioned before, our QS, FS and QSFS policies are based on the modified threshold policy proposed in [1], but are different since we use PS+Priority queueing instead of PS+FCFS queueing. If we compare the simulation results of our new basic QS, FS and QSFS policies with results of the modified threshold policy proposed in [1] we can easily observe that our new switching policies improve on the modified threshold policy by around $17 \%$ in terms of response time. 


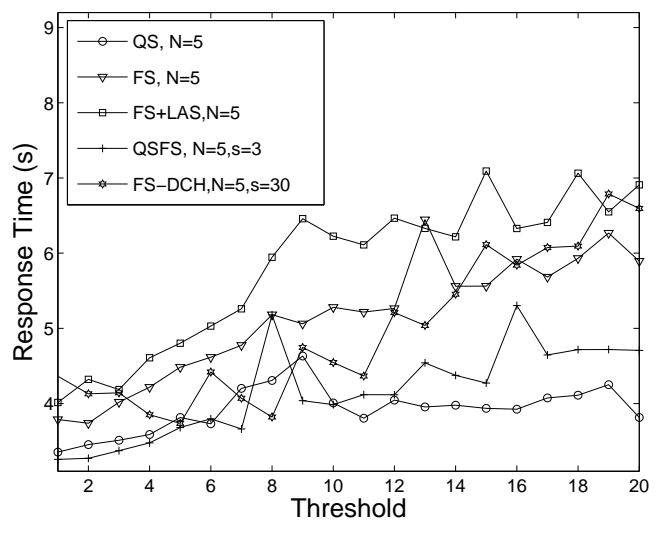

(a)

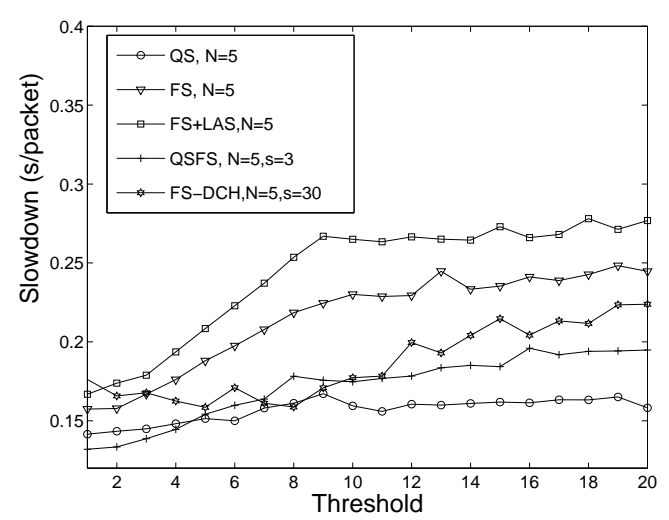

(b)

Figure 4: Comparison of different policies in terms of response time and slowdown metrics for $N_{t c p}=5, F S_{a v g}=30$ kbytes and $N_{d c h}=1$.

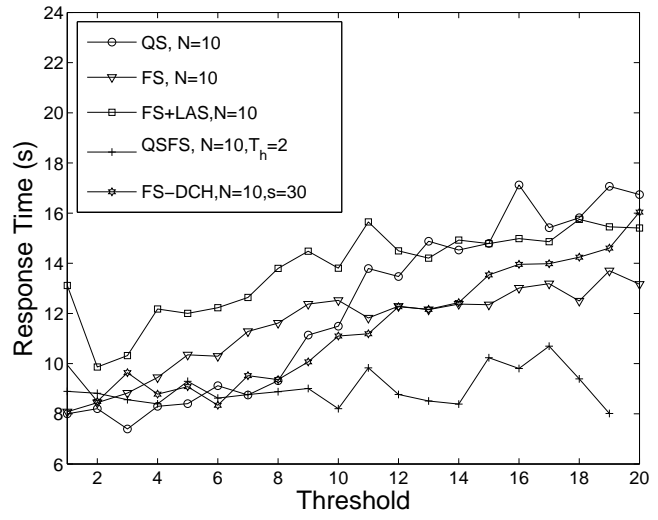

(a)

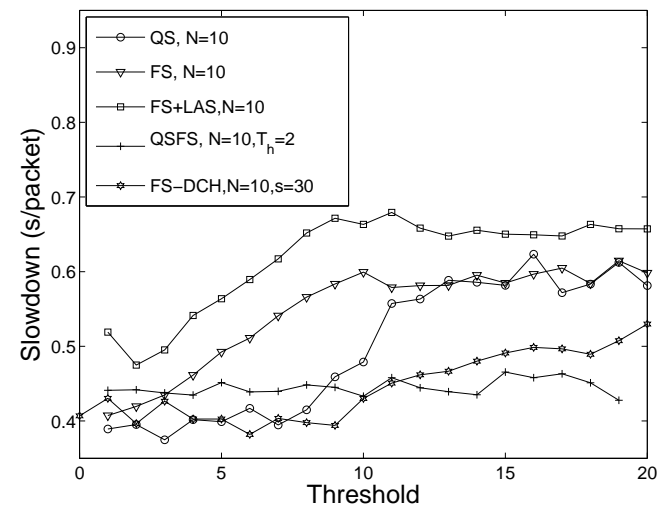

(b)

Figure 5: Comparison of different policies in terms of response time and slowdown metrics for $N_{t c p}=10, F S_{a v g}=30$ kbytes and $N_{d c h}=1$. 


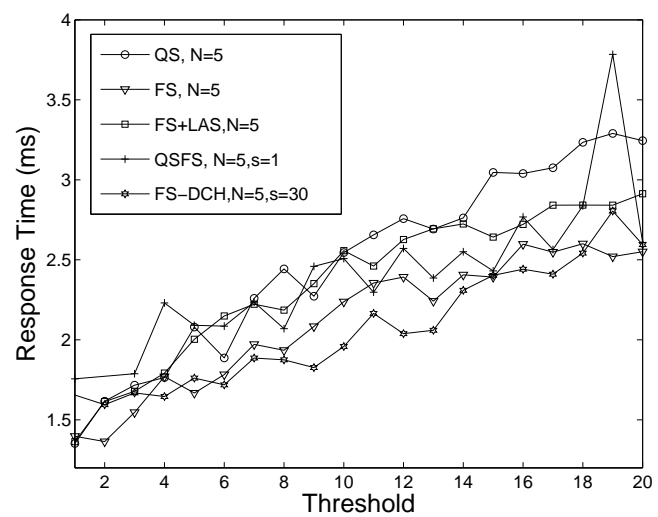

(a)

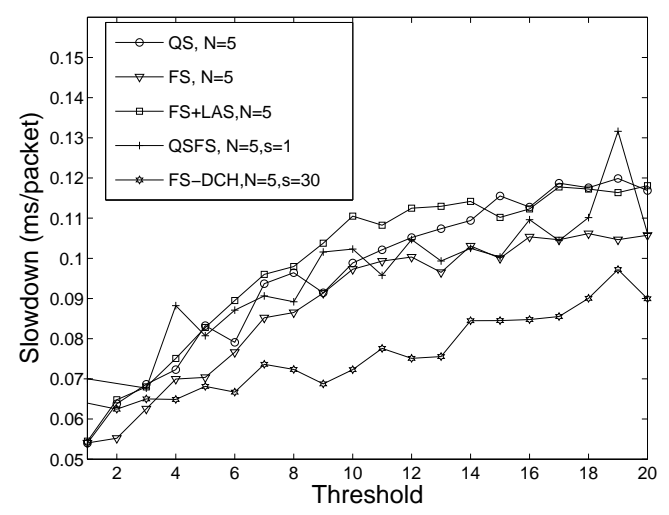

(b)

Figure 6: Comparison of different policies in terms of response time and slowdown metrics for $N_{t c p}=5, F S_{a v g}=30$ kbytes and $N_{d c h}=2$.

\subsection{Effect of Number of DCHs}

Here we discuss about performance of different policies when the number of dedicated channels is increased to 2. In Figure 6a) we observe that FS-DCH again outperforms other policies in terms of response time. Other policies perform comparably. In Figure 6 b), we observe that slowdown follows the same trend as that of response time.

\section{Conclusion}

In this paper, we have proposed several scalable channel switching policies for packet data transmission on UMTS downlink. The basic threshold based policies QS, FS and QSFS are based on an extension of the modified threshold policy in [1]. In our policies, we use PS+Priority and LAS+Priority queue systems for FACH and DCH channels instead of PS+FCFS queue system used in [1]. Simulation results show that our new basic switching policies improve on the modified threshold policy in [1] by around $17 \%$ in response time metrics. We have further proposed a new and improved crosslayer channel switching policy that we call FS-DCH (at-least flow-size threshold on DCH) policy. FS-DCH is a biased policy that improves the performance of TCP flows by giving priority to short flows on the fast DCH channel. Results obtained from extensive simulations show that for a given simulation set-up of $N_{d c h}=1$ and $F S_{\text {avg }}=30$ kbytes, FS-DCH performs better than the basic QS, FS and QSFS policies, when the number of TCP connections $N_{t c p}$ is low. For example for $N_{t c p}=2$ and 3, FS-DCH gives a significant average improvement of $30 \%$ to $36 \%$, respectively, over all other policies, in terms of response time. However, with higher number of TCP connections $N_{t c p}$, the best performing policies are the ones that are queue size based i.e., QS and QSFS policies. They give an 
average improvement in response time of $18 \%$ and $21 \%$, respectively. One can also observe that the best performing policies in terms of response time, also outperform in terms of slowdown.

\begin{tabular}{|l|c|c|}
\hline$N_{t c p}$ & $\begin{array}{c}\text { Best Performing } \\
\text { Policy }\end{array}$ & $\begin{array}{c}\text { Percentage gain } \\
\text { in Response Time }\end{array}$ \\
\hline 2 & FS-DCH & $30 \%$ \\
3 & FS-DCH & $36 \%$ \\
5 & QS & $18 \%$ \\
10 & QSFS & $21 \%$ \\
\hline
\end{tabular}

Table 1: Best performing policies for different values of $N_{t c p}, N_{d c h}=1$ and $F S_{a v g}=30$ kbytes

It can also be concluded that there is no single policy that can be termed as an overall best performer and for different number of TCP connections, different policies exhibit improved performance over other policies. Table I shows the best performing policies in terms of response time, under different number of TCP connections.

\section{References}

[1] B. J. Prabhu, E. Altman, K. Avrachenkov, J. A. Dominguez, A Simulation Study of TCP Performance over UMTS Downlink, Proc. IEEE VTC-Fall, Orlando, USA, Oct. 2003.

[2] H. Holma, A. Toskala, WCDMA for UMTS, Rev. Ed., Wiley, 2001.

[3] C. Anderson, J. Soderberg, Channel-Type Switching from a Common Channel to A Dedicated Channel based on Common Channel Load, US Patent no. US-6,519,461. 2003.

[4] Y. Ohta, K. Kawahara, T. Ikenaga, Y. Oie, Performance Evaluation of Channel Switching Scheme for Packet Data Transmission in Radio Network Controller, Proc. Networking, Berlin, 2002.

[5] Roy F. Quick, Jr., Random Access Communications Channel for Data Services, US Patent no. US-5,673,259. 2003.

[6] A. Floberg, Channel type switching in WCDMA, Technical Report, April 1999.

[7] K. Avrachenkov, U. Ayesta, P. Brown, J. Blanton, Differentiation between Short and Long TCP flows: Predictability of the response time, Proc. Infocom, Hong Kong, March 2004.

[8] The Network Simulator - ns-2, http://www.isi.edu/nsnam/ns

[9] A. Weirman and M. Harchol-Balter, Classifying scheduling policies with respect to unfairness in M/GI/l, Proc. ACM Sigmetrics, 2003.

[10] A. Weirman and M. Harchol-Balter, Classifying Scheduling Policies with Respect to Higher Moments of Conditional Response Time, Proc. ACM Sigmetrics, 2005. 


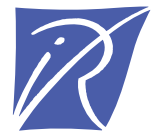

\section{Unité de recherche INRIA Sophia Antipolis 2004, route des Lucioles - BP 93 - 06902 Sophia Antipolis Cedex (France)}

Unité de recherche INRIA Futurs : Parc Club Orsay Université - ZAC des Vignes 4, rue Jacques Monod - 91893 ORSAY Cedex (France) Unité de recherche INRIA Lorraine : LORIA, Technopôle de Nancy-Brabois - Campus scientifique 615, rue du Jardin Botanique - BP 101 - 54602 Villers-lès-Nancy Cedex (France)

Unité de recherche INRIA Rennes : IRISA, Campus universitaire de Beaulieu - 35042 Rennes Cedex (France)

Unité de recherche INRIA Rhône-Alpes : 655, avenue de l'Europe - 38334 Montbonnot Saint-Ismier (France)

Unité de recherche INRIA Rocquencourt : Domaine de Voluceau - Rocquencourt - BP 105 - 78153 Le Chesnay Cedex (France) 


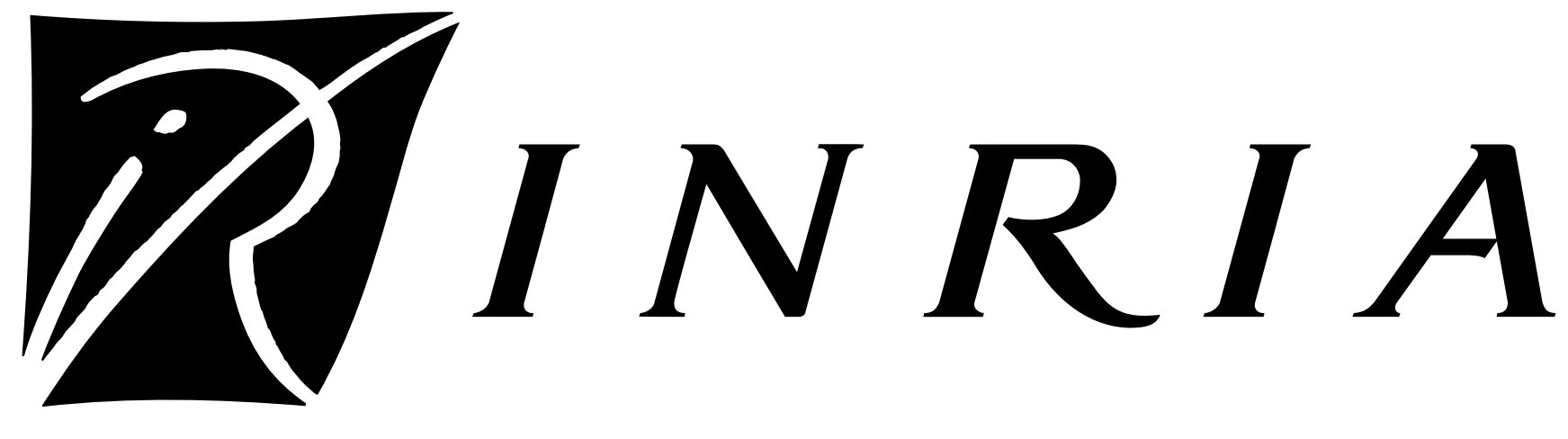


